\title{
Comparison of Yarrowia lipolytica and Pichia pastoris Cellular Response to Different Agents of Oxidative Stress
}

\author{
Marlene Lopes • Manuel Mota • Isabel Belo
}

Received: 13 November 2012 / Accepted: 18 March 2013 /

Published online: 2 April 2013

(C) Springer Science+Business Media New York 2013

\begin{abstract}
Yeast cells exposed to adverse conditions employ a number of defense mechanisms in order to respond effectively to the stress effects of reactive oxygen species. In this work, the cellular response of Yarrowia lipolytica and Pichia pastoris to the exposure to the ROSinducing agents' paraquat, hydrogen peroxide, and increased air pressure was analyzed. Yeast cells at exponential phase were exposed for $3 \mathrm{~h}$ to $1 \mathrm{mM}$ paraquat, to $50 \mathrm{mM} \mathrm{H}_{2} \mathrm{O}_{2}$, or to increased air pressure of 3 or 5 bar. For both strains, the cellular viability loss and lipid peroxidation was lower for the cells exposed to increased air pressure than for those exposed to chemical oxidants. The glutathione induction occurred only in Y. lipolytica strain and reached the highest level as a response to PQ exposure. In general, antioxidant enzymes were more expressed in Y. lipolytica than in P. pastoris. The enzyme superoxide dismutase was induced in both strains under all the oxidant conditions but was dependent on the cellular growth phase, being undetectable in non-growing cells, whereas glutathione reductase was more induced in those conditions. Hydrogen peroxide was the most efficient inducer of catalase. Both yeast cultures underwent no cellular growth inhibition with increased air pressure, indicating that these yeast species were able to adapt to the oxidative stressful environment.
\end{abstract}

Keywords Oxidative stress · Antioxidant enzymes - Increased air pressure Yarrowia lipolytica Pichia pastoris

\section{Introduction}

All aerobic organisms use molecular oxygen for respiration and oxidation of nutrients to obtain energy efficiently. During the reduction of molecular oxygen to water through

M. Lopes $\cdot$ M. Mota $(\bowtie) \cdot$ I. Belo

IBB - Institute for Biotechnology and Bioengineering, Centre of Biological Engineering, University of Minho, Campus de Gualtar, 4710-057 Braga, Portugal

e-mail:mmota@deb.uminho.pt

M. Lopes

e-mail: marlenelopes@deb.uminho.pt

I. Belo

e-mail: ibelo@deb.uminho.pt 
acceptance of four electrons, aerobic microorganisms have to face the toxic effects of oxygen, once active oxygen species such as superoxide anion radical $\left(\mathrm{O}_{2}{ }^{-}\right)$, hydrogen peroxide $\left(\mathrm{H}_{2} \mathrm{O}_{2}\right)$, and hydroxyl radicals are generated. These reactive oxygen species (ROS) are highly noxious to all biological molecules, including DNA, proteins, and lipids [1].

The rise of total air pressure leads to an increase of oxygen partial pressure, which generates ROS [2]. Pro-oxidant compounds in the culture medium, such as paraquat (PQ) and hydrogen peroxide also have the capacity to generate intracellular ROS.

Paraquat (1,1'-dimethyl-4,4'-bipyridylium dichloride) is a quaternary nitrogen herbicide and in the presence of a sufficient supply of reducing equivalents, repeated cycles of herbicide reduction and reoxidation can occur, producing large amounts of reactive oxygen species, oxidative stress, and lipid peroxidation [3].

The addition of exogenous hydrogen peroxide to microbial cultures may result in oxidative stress. The adaptation to this radical requires protein synthesis and the expression of at least 21 proteins increased following $\mathrm{H}_{2} \mathrm{O}_{2}$ adaptation [4].

The inadequate mixing and mass transfer that cells might face in an industrial submerged culture process may expose yeast cells to variations in dissolved oxygen, including transient exposure to regions of high dissolved oxygen, leading to oxidative stress. Despite the industrial significance, few studies were performed to simulate the impact of oxidative stress incurred by exposure to increased oxygen partial pressure and elevated dissolved oxygen on Saccharomyces cerevisiae [5], Yarrowia lipolytica [6], and Kluyveromyces marxianus [7] cultures.

Cells possess several defensive enzymatic (such as superoxide dismutase, catalase, and glutathione reductase) and nonenzymatic (such as glutathione) mechanisms to protect their cellular constituents and maintain cellular redox state.

Y. lipolytica, a non-conventional yeast, is a strictly aerobic microorganism with the ability to grown in a variety of hydrophobic compounds including alkanes, oils, and fatty acids, as well as in olive mill wastewater. It is most used as an adequate model for dimorphism studies in yeasts and is capable of producing important industrial metabolites, such as lipase, citric acid, biosurfactants, aroma, and microbial lipids [8]. The methylotrophic Pichia pastoris can grow to high-cell density and has the potential for high-level expression of recombinant proteins, such as $\beta$-glucosidase [9], $\beta$-galactosidase [10], and frutalin [11], among others. P. pastoris expression system offers the advantages of having a strong methanol-induced promoter (alcohol oxidase 1 promoter) and the capability of performing many eukaryotic posttranslational modifications, such as glycosylation, disulfide bond formation, and proteolytic processing [12].

To learn more about the response of these yeast strains to oxidative stress, the effect of different ROS-generating agents on cell viability and on the induction of antioxidant enzymes were studied. The content of malondialdehyde (MDA) and reduced glutathione (GSH) and the induction of antioxidant enzymes (superoxide dismutase (SOD), catalase, glutathione reductase (GR)) in response to $\mathrm{H}_{2} \mathrm{O}_{2}$, PQ and increased air pressure were investigated. To our knowledge this is the first study concerning strains of $P$. pastoris and $Y$. lipolytica and their interaction with the pro-oxidant agents. Moreover, this is the first time that these yeast strains were exposed to oxidant agents in a non-respiratory medium (phosphate buffered saline (PBS) buffer) and a more detailed analyses of cellular antioxidant indicators was performed. 


\title{
Material and Methods
}

\author{
Strains and Media
}

Y. lipolytica W29 (ATCC 20460) and P. pastoris CBS 2612 were grown in yeast peptone dextrose (YPD) (10 g/L yeast extract, $10 \mathrm{~g} / \mathrm{L}$ peptone, $20 \mathrm{~g} / \mathrm{L}$ glucose) medium. The yeast strains were maintained in YPD agar plates and stored at $4{ }^{\circ} \mathrm{C}$ to a maximum of 1 month.

\section{Oxidants Treatment}

Yeasts cells were precultured during $9 \mathrm{~h}$ (exponential phase) in 250-mL Erlenmeyer flasks filled with $100 \mathrm{~mL}$ of YPD medium. Cells were then harvested by centrifugation, washed with PBS buffer, and resuspended in $400 \mathrm{~mL}$ of PBS buffer or YPD medium. In the experiments with chemical oxidants, PQ and $\mathrm{H}_{2} \mathrm{O}_{2}$ were added at nonlethal final concentration of 1 and $50 \mathrm{mM}$, respectively. The exposure to increased air pressure was performed in a $600-\mathrm{mL}$ pressurized bioreactor (stainless steel stirred tank, PARR 4563, Parr Instruments, USA) under 3 or 5 bar of air pressure at $400 \mathrm{rpm}$ and $1 \mathrm{vvm}$ (volume of air/volume of liquid/minute) of aeration.

\section{Batch Growth Under Increased Air Pressure}

Yeasts cells were pre-grown overnight in 250-mL Erlenmeyer flasks with $100 \mathrm{~mL}$ of YPD at $140 \mathrm{rpm}$ and at $27^{\circ} \mathrm{C}$ (Y. lipolytica) or $30^{\circ} \mathrm{C}$ (P. pastoris). Batch cultivations were carried out in the pressurized bioreactor, with $400 \mathrm{~mL}$ of each culture, at 27 or $30{ }^{\circ} \mathrm{C}$ and $400 \mathrm{rpm}$. Compressed air was continuously sparged into the culture at an aeration rate of $1 \mathrm{vvm}$. The values of air absolute pressure studied were 1, 3, and 5 bar. Details of the pressurized bioreactor are described elsewhere [6].

\section{Analytical Methods}

Yeasts samples were collected after $3 \mathrm{~h}$ of exposure to oxidants for analysis of cell viability, MDA, and GSH content and antioxidant enzymes activity. Cell viability was estimated by the methylene blue staining method [13]. Antioxidant enzymes were measured after cell disruption and dialysis of cell extracts, as described elsewhere [14]. Catalase was assayed using the method described by Beers and Sizer [15], SOD was quantified by the method of Marklund and Marklund [16] and glutathione reductase was analyzed according to the procedure described by Smith et al. [17]. MDA was measured by the method of thiobarbituric acid reactive species as described by Espindola et al. [18]. GSH was quantified in the neutralized extracts using DTNB reagent according to the procedure described by Jamnik and Raspor [19].

Samples from the batch cultures in the pressurized bioreactor were collected for analysis of cell concentration (optical density at $600 \mathrm{~nm}$ and converted to dry cell weight per liter) and glucose consumption. Glucose was quantified by HPLC with a Metacarb $67 \mathrm{H}$ column (Varian, Palo Alto, CA) and a RI detector. The eluent was $\mathrm{H}_{2} \mathrm{SO}_{4} 0.005 \mathrm{~mol} / \mathrm{L}$ at $0.5 \mathrm{~mL} / \mathrm{min}$ and the column temperature was $60{ }^{\circ} \mathrm{C}$. Results are presented as the average \pm standard deviation of three independent experiments. 


\title{
Results and Discussion
}

\author{
Cell Viability
}

The effect of three different oxidative stress inductors, PQ, $\mathrm{H}_{2} \mathrm{O}_{2}$, and hyperbaric air, on yeast cell viability was tested. The experiments were conducted with cells at the exponential phase since these cells are metabolically more active than stationary phase cells and were likely to be more susceptible to the possible lethality of aerobic stressors [20]. For exposure assays the yeast cells were resuspended in two different culture media, in order to analyze the antioxidant response of respiring and non-respiring cells.

The presence of a carbon source allowed the cells to grow during the oxidant exposure, and led to more active and viable cells. In the experiments with YPD medium, there was no significant difference on the cellular viability between strains independent of the stressors (Table 1). However, the exposure to oxidants agents in PBS buffer demonstrated that the P. pastoris cells were more susceptible than the Y. lipolytica. Hassan and Fridovich [20] also observed that the Escherichia coli cells were strikingly less sensitive to PQ in complex medium than in minimal medium.

When cells of Y. lipolytica were exposed to $\mathrm{H}_{2} \mathrm{O}_{2}$ and PQ in PBS buffer, respectively, 87 and $90 \%$ of the cells survived after $3 \mathrm{~h}$ of exposure, which shows that the strain is quite resistant to these oxidant agents. The exposure to hyperbaric air leads to a minor decrease in cell viability, indicating that the oxidative stress imposed by the partial oxygen pressure is less deleterious than the other stressors. The P. pastoris cells were more susceptible to the oxidative stress than the other strain, mainly in PBS buffer. When Pichia cells were subjected to the PQ and $\mathrm{H}_{2} \mathrm{O}_{2}$ treatment about 36 and $42 \%$ were killed, respectively. In turn, there was 78 and $77 \%$ of viable cells in treatments under 3 and 5 bar of air, respectively. Pinheiro et al. [21] reported that $K$. marxianus cells respond better to the PQ than to the $\mathrm{H}_{2} \mathrm{O}_{2}$ exposure during $24 \mathrm{~h}$ of growth. Also, cell viability was higher for the cells growing under increase air pressure up to 6 bar than for those cells exposed to chemical oxidants. Data suggest that different organisms respond differently to ROS and that they use different defense mechanisms against those substances, namely the nonprotein thiol GSH and a variety of antioxidant enzymes.

\section{Antioxidant Markers}

Lipid peroxides are unstable indicators of oxidative stress in cells that decompose to form more complex and reactive compounds such as MDA, a natural byproduct of lipid peroxidation. Regardless of the yeast strain, the exposure to oxidant agents leads to MDA production. This production was more pronounced with the PQ treatment (Fig. 1). It has been proposed that the cyclic reduction-oxidation of PQ initiates lipid peroxidation through

Table 1 Cell viability (\%), defined as the ratio of final and initial viability, of two yeasts strains in exponential phase of growth, resuspended in PBS buffer and YPD medium, exposed to different oxidants for 3 h. Values are average \pm standard deviation of three independent experiments

\begin{tabular}{|c|c|c|c|c|c|c|c|c|}
\hline & \multicolumn{4}{|c|}{ PBS buffer } & \multicolumn{4}{|c|}{ YPD medium } \\
\hline & $\mathrm{H}_{2} \mathrm{O}_{2}$ & PQ & 3 bar & 5 bar & $\mathrm{H}_{2} \mathrm{O}_{2}$ & PQ & 3 bar & 5 bar \\
\hline Y. lipolytica W29 & $87 \pm 11$ & $90 \pm 10$ & $95 \pm 12$ & $95 \pm 13$ & $92 \pm 15$ & $94 \pm 13$ & $97 \pm 14$ & $96 \pm 15$ \\
\hline P. pastoris CBS 2612 & $58 \pm 8$ & $64 \pm 10$ & $78 \pm 12$ & $77 \pm 12$ & $90 \pm 14$ & $93 \pm 15$ & $97 \pm 14$ & $96 \pm 14$ \\
\hline
\end{tabular}


subsequent superoxide radical and singlet oxygen intermediates. The chemical cascades leading to the reduction of PQ and the generation of free radicals and lipid peroxidation are the main factors that lead to mitochondrial damage [22].

The MDA content in cells resuspended in PBS buffer and exposed to PQ was three- and tenfold higher than in the experiments under 5 bar for Y. lipolytica and P. pastoris cells, respectively. The exposure of cells in YPD medium to $\mathrm{H}_{2} \mathrm{O}_{2}$ led to a six and twofold enhancement in MDA production compared to 5 bar of air pressure for Y. lipolytica and $P$. pastoris cells, respectively. Interestingly, it seems that lipid peroxidation with hydrogen peroxide is minimized, being $P$. pastoris less sensitive to the stress caused by $\mathrm{H}_{2} \mathrm{O}_{2}$. Thus, it is reasonable to conclude that the stress caused by an increase of partial oxygen pressure did not lead to membrane destructive processes.

It seems that there was no direct relation between the medium composition and the induction of lipid peroxidation. Although the Y. lipolytica strain exposed to PQ showed a 2.4-fold increase in MDA content when the cells were in PBS buffer compared to YPD medium, the addition of the $\mathrm{H}_{2} \mathrm{O}_{2}$ led to a threefold enhancement of MDA in PBS buffer.

The involvement of oxidative mechanisms to mediate the damaging effects of oxidant agents tested has been first shown by the analysis of GSH. According to our results, only the Y. lipolytica strain has shown the capacity to induce GSH production in the experimental conditions (Fig. 2). It is likely that P. pastoris possesses other defense mechanisms more important than GSH in antioxidant response to stressors, such as antioxidant enzymes SOD or catalase. Also, this low GSH production in Pichia might be responsible for the lower-cell viability in PBS medium observed for this yeast as compared to Y. lipolytica.

The exposure of Y. lipolytica cells to PQ led to a 30-fold enhancement on GSH induction comparatively to the experiments conducted with hydrogen peroxide. Also, GSH was more induced with air pressure than with $\mathrm{H}_{2} \mathrm{O}_{2}$. The cells exposed to hydrogen peroxide showed a minor GSH induction. The GSH content in experiments with $\mathrm{H}_{2} \mathrm{O}_{2}$ was 30- and 9-fold smaller than the value obtained with PQ and 5 bar of air pressure. Among the oxidative stress agents, PQ is a thiol-oxidizing agent resulting in fast oxidation of GSH to oxidized glutathione (GSSG). The comparison of GSH in cells treated with PQ and increased air pressure indicates that the thiol oxidation should be small up to 5 bar of air pressure.

Glutathione is an essential reductant during normal metabolic processes in yeasts. Grant et al. [23] demonstrated that a $S$. cerevisiae strain which lacks a functional copy of the GSH 1 gene

a

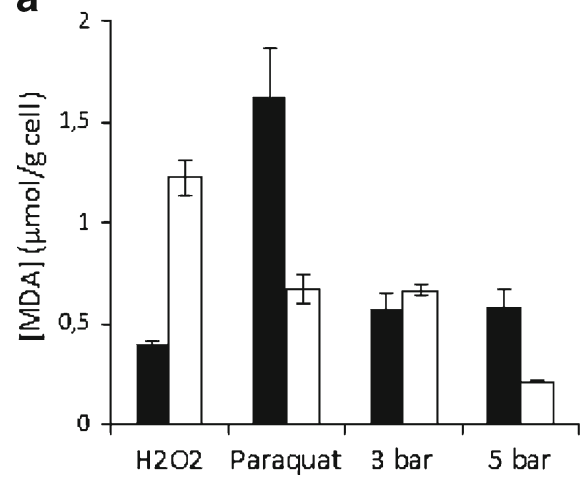

b

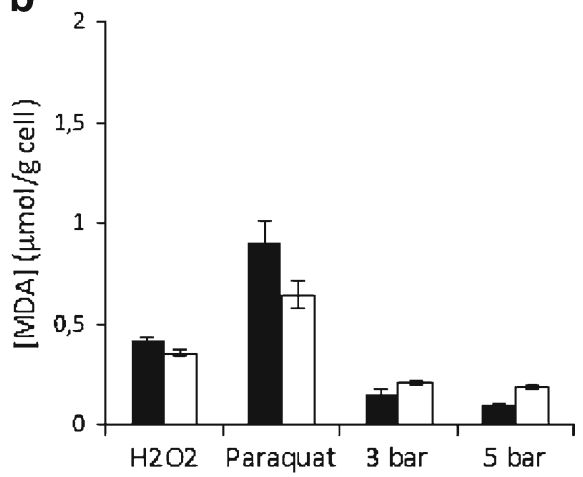

Fig. 1 MDA content of a Y. lipolytica W29 and b P. pastoris CBS 2612 cells in exponential phase of growth, resuspended in PBS buffer (black bars) and YPD medium (white bars) and exposed to different oxidants. Values are average \pm standard deviation of three independent experiments 


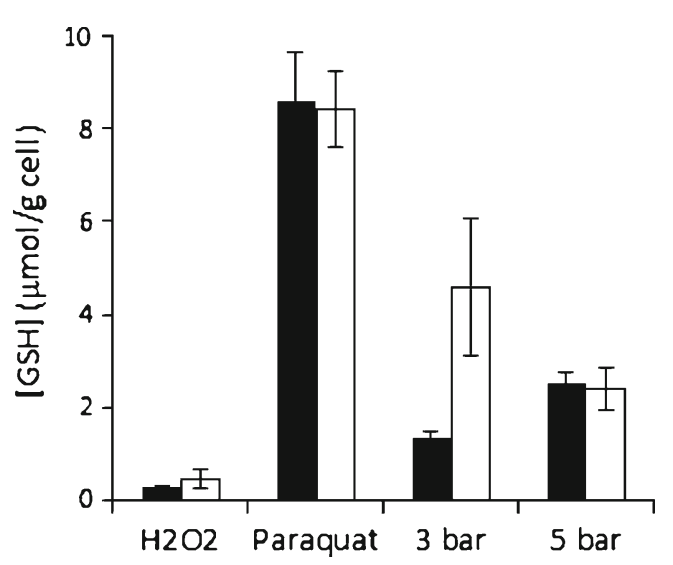

Fig. 2 GSH content of Y. lipolytica W29 cells in exponential phase of growth resuspended in PBS buffer (black bars) and YPD medium (white bars) and exposed to different oxidants. Values are average \pm standard deviation of three independent experiments

is hypersensitive to the peroxides and the oxidative stress conditions induced by the $\mathrm{H}_{2} \mathrm{O}_{2}$. Izawa et al. [24] demonstrated that intracellular GSH played an important role in the stress response to $\mathrm{H}_{2} \mathrm{O}_{2}$ in $\mathrm{S}$. cerevisiae using glutathione-depleting agents and a glutathione-deficient mutant. This could explain the major decrease of yeast cells viability exposed to $\mathrm{H}_{2} \mathrm{O}_{2}$ comparatively to the others stressors agents, as reported above.

One of the principal antioxidant enzymes is superoxide dismutase that is involved in the dismutation of superoxide anions to dioxygen and hydrogen peroxide. In the experiments with yeast cells resuspended in PBS buffer, both strains were unable to induce the SOD enzyme. This result may suggest that only in the presence of a respiratory carbon source, the yeast strains used on this work have the capacity to induce this enzyme. The general non-induction of SOD observed after oxidant agents treatment in PBS buffer could also be the result of oxidative damage of this enzyme, because it is known to be repressed by various peroxides. This probably reflects the decreased ability of the cells to adapt efficiently to the oxidative stress. Whereas SOD is considered to be an essential antioxidant enzyme, at the same time it can have prooxidant effects in vivo [25], and thus, SOD inactivation can also be the result of a cellular defense mechanism. Hassan and Fridovich [20] found that E. coli cells responded to PQ by increasing their content of SOD in the presence of yeast extract, but not in its absence, leading to the assumption that yeast extract might have exerted its effect by eliciting the SOD biosynthesis.

Although there were differences in the other defensive mechanisms between the two yeasts tested (e.g., GSH and catalase), SOD induction was quite similar for both strains, with each oxidant treatment (Fig. 3). This may be due to the fact that this enzyme is one of the primarily induced antioxidant mechanisms involved in stress defense.

The experiments under air pressure up to 5 bar produced a clear increase in SOD activity compared to the exposure to $\mathrm{H}_{2} \mathrm{O}_{2}(69 \%$ high for Y. lipolytica and $65 \%$ high for P. pastoris) and PQ (57\% high for Y. lipolytica and $65 \%$ high for P. pastoris). This high SOD activity in the experiments under 3 and 5 bar of air pressure may explain the higher cell viability of the both strains. Previous work demonstrated that the increase of total air pressure up to 6 bar leads to an enhancement of intracellular SOD of Y. lipolytica W29 [6]. The importance of yeasts superoxide dismutase enzyme on triggering ROS generation by oxygen was also proved by Lushchak et al. [26], who observed that $S$. cerevisiae strains carrying mutations in $S O D 1$ and SOD 2 genes were hypersensitive to oxygen, causing slow aerobic growth. 


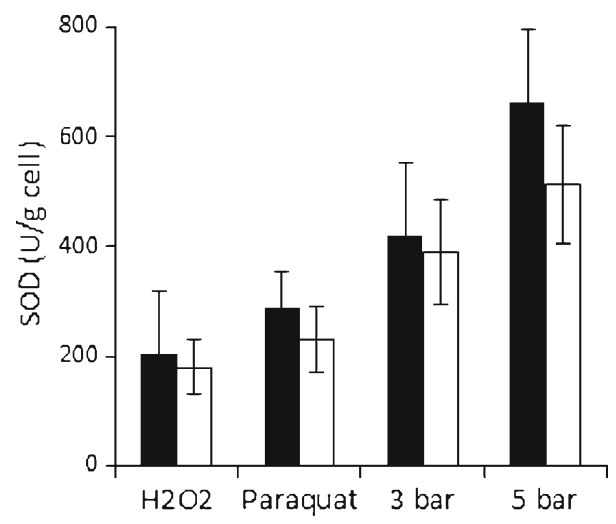

Fig. 3 Superoxide dismutase specific activity of Y. lipolytica W29 (black bars) and P. pastoris CBS 2612 (white bars) cells in exponential phase of growth resuspended in YPD medium and exposed to different oxidants. Values are average \pm standard deviation of three independent experiments

The low SOD activity in yeast cells incubated with $50 \mathrm{mM} \mathrm{H} \mathrm{H}_{2} \mathrm{O}_{2}$ suggests that this enzyme does not participate in the cellular acclimatization to drastic oxidative $\mathrm{H}_{2} \mathrm{O}_{2}$-induced challenges. Abbeg et al. [27] observed only a slight induction of SOD with the addition of $\mathrm{H}_{2} \mathrm{O}_{2}$ to the various Candida strains medium and Biryukova et al. [28] found a slight induction of SOD with the pretreatment of $Y$. lipolytica cells with $0.3 \mathrm{mM} \mathrm{H}_{2} \mathrm{O}_{2}$.

The activation of catalase observed following each oxidant stressors treatment, is one of the most common cellular responses to redox alterations, because this enzyme is easily induced by a wide range of stimuli often related to the energy status of the cell [29].

In general, the oxidant treatments with cells resuspended in PBS buffer showed a higher catalase activity compared to those with YPD medium (Fig. 4). A 1.7-, 2.9-, 5-, and 3.8-fold enhancement in enzyme activity was obtained when $Y$. lipolytica cells resuspended in PBS buffer were exposed to $\mathrm{H}_{2} \mathrm{O}_{2}, \mathrm{PQ}, 3$ and 5 bar of air pressure, respectively, compared to the activities with YPD medium. The glucose concentration can exert a negative pressure on the catalase activity of Y. lipolytica W29. In fact, Braconi et al. [29] reported the same effect in the early phase of $S$. cerevisiae growing in high glucose concentration medium. The

a

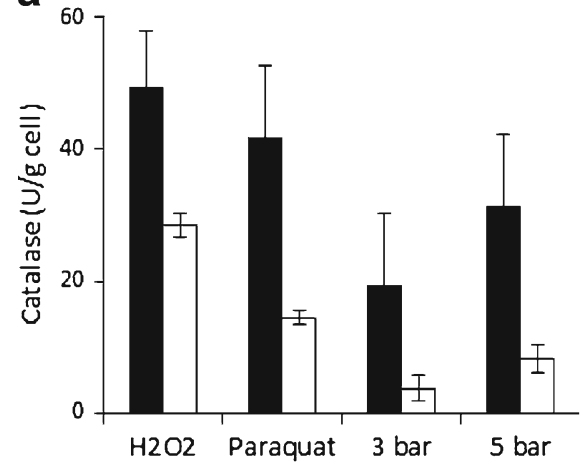

b

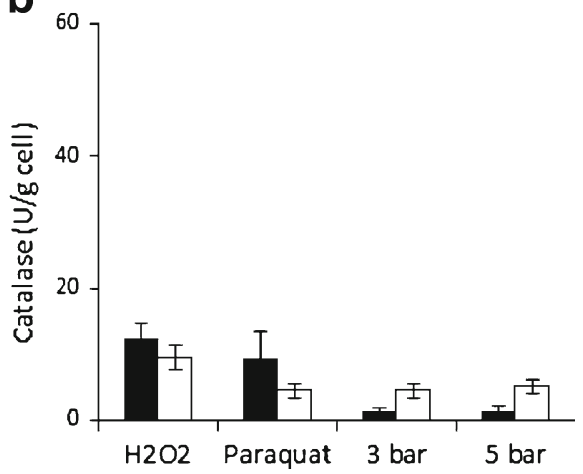

Fig. 4 Catalase specific activity of a Y. lipolytica W29 and b P. pastoris CBS 2612 cells in exponential phase of growth, resuspended in PBS buffer (black bars) and YPD medium (white bars) and exposed to different oxidants. Values are average \pm standard deviation of three independent experiments 
differences found in the experiments with $P$. pastoris were not so significant. On the other hand, as both yeast cells resuspended in PBS buffer were not capable to induce SOD, it can be concluded that both SOD and catalase operate in concert to protect cells from oxidative stress. Such relationship can be connected with the compensatory response of the yeast cells to maintain the steady state level of ROS inside the cells [30]. Semchyshyn and Lozinska [31] observed that hydrogen peroxide increased catalase activity in SOD-deficient mutant.

When comparing the two yeasts, it was observed that Yarrowia showed a higher capacity to induce catalase after exposure to oxidant agents. A 4-, 16-, and 23-fold enhancement was obtained in Y. lipolytica with $\mathrm{H}_{2} \mathrm{O}_{2}$ and PQ, 3 and 5 bar of air pressure, respectively, compared to the activities obtained with $P$. pastoris.

Particular attention should be paid to $\mathrm{H}_{2} \mathrm{O}_{2}$, the stress treatment that leads to a higher catalase synthesis in both yeast strains, regardless of the medium where yeast cells were resuspended. The exposure of Y. lipolytica cells to $\mathrm{H}_{2} \mathrm{O}_{2}$, resuspended in YPD medium, led to a 1.9-, 7.5-, and 3.5-fold increase in catalase activity as compared to PQ, 3 and 5 bar of air pressure treatments. The enzyme activity of $P$. pastoris cells resuspended in PBS medium and treated with $\mathrm{H}_{2} \mathrm{O}_{2}$ was 1.3, 9.9, and 8.9 times higher than that obtained in exposures to PQ, 3 and 5 bar of air pressure, respectively. Other authors have demonstrated that the treatment with $\mathrm{H}_{2} \mathrm{O}_{2}$ induced increased levels of catalase activity in P. pastoris [17], Candida albicans [32], various Candida strains [27], Aspergillus niger [33], Y. lipolytica [28], and S. cerevisiae [34].

The enzyme glutathione reductase is involved in the glutathione recycling system. This enzyme enables the cell to sustain adequate levels of cellular GSH, once it is primarily responsible for the reduction of GSSG to reduced glutathione at the expense of nicotinamide adenine dinucleotide phosphate (NADPH).

Both yeast strains were able to induce glutathione reductase (Fig. 5). However, the GR was considerably smaller than the SOD and catalase activities, suggesting that glutathione reductase plays a minor role on antioxidant defense against the agents tested. On the other hand, as the process of redox-cycling depletes intracellular NADPH (cofactor for glutathione reductase), the enzyme activity was likely to be affected by oxidative stress. It seems that there is a relation between the medium composition and the glutathione reductase induction once the cells resuspended in PBS buffer showed higher activity, whatever the oxidant agent tested and yeast strain may be.
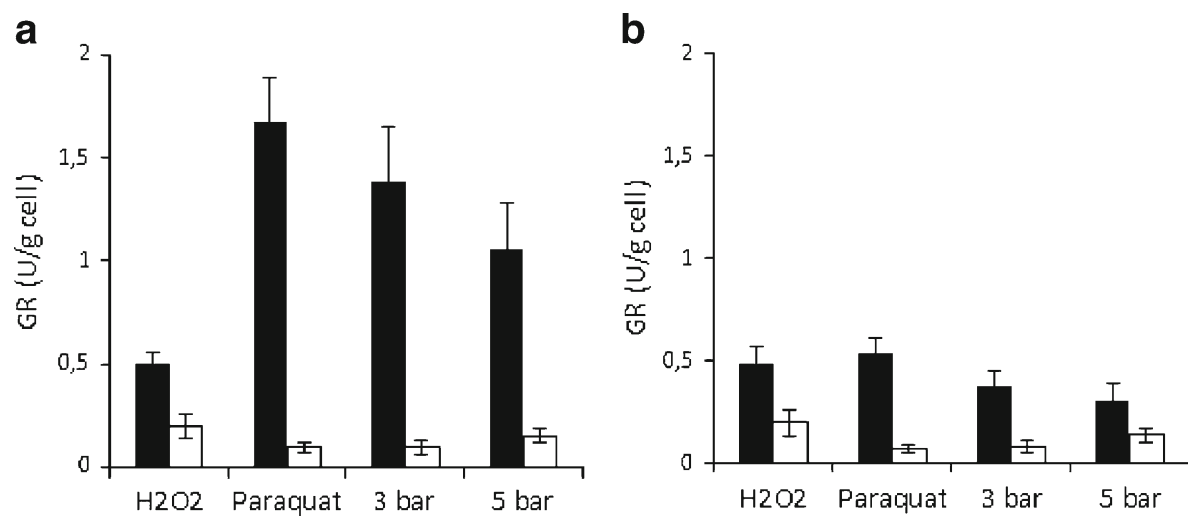

Fig. 5 Glutathione reductase specific activity of a Y. lipolytica W29 and b P. pastoris CBS 2612 cells in exponential phase of growth, resuspended in PBS buffer (black bars) and YPD medium (white bars) and exposed to different oxidants. Values are average \pm standard deviation of three independent experiments 
In all the experiments with $P$. pastoris cells there was no significant difference in GR enzyme activity with any of the pro-oxidant agents. However, with the Y. lipolytica, cells treated with $\mathrm{H}_{2} \mathrm{O}_{2}$ showed less glutathione reductase activity. A 3.3-fold enhancement in enzyme activity was observed in the treatment with PQ compared to $\mathrm{H}_{2} \mathrm{O}_{2}$ exposure. It was not surprising that the higher activity of this enzyme was found for the agents that also lead to a more pronounced induction of GSH (PQ and increased air pressure) once this enzyme participates in the reduction of GSSG to GSH in the presence of NADPH.

\section{Growth Under Increased Air Pressure}

Since Y. lipolytica and P. pastoris showed to be able to adapt to pressures of 3 and 5 bar and it was showed that antioxidant defense mechanisms are induced under these conditions, batch cultures of the yeast strains under increased air pressure were performed to validate the resistance of the yeasts under this stress condition. Typical batch growth and glucose consumption profiles are shown in Fig. 6. Both strains were able to grow for $24 \mathrm{~h}$ under air pressure values 5 -fold higher than the atmospheric pressure.

Regardless of the yeast strain, the rise of total air pressure from 1 to 3 and 5 bar led to an increase in the final cell dry weight. 3.3-fold and 1.9-fold improvement in biomass production was obtained with the increase of air pressure up to 5 bar compared to $1 \mathrm{bar}$, for Y. lipolytica and P. pastoris, respectively. Among the yeast strains studied, the highest biomass yield was obtained with 5 bar of air pressure. An improvement in biomass yield of Y. lipolytica and P. pastoris cultures from 0.3 to 1.1 mass of cells per mass of glucose and from 0.3 to 0.6 mass of cells per mass of glucose, respectively, was achieved with increase air pressure up to 5 bar. The values of specific growth rate obtained at 1 and 5 bar were 0.18 and $0.28 \mathrm{~h}^{-1}$, respectively. The rise of air pressure from 1 to 5 bar led to a 1.6-fold improvement in specific growth rate. However, no significant differences were obtained on growth rates of $P$. pastoris when the pressure varies from 1 to 5 bar $\left(0.27-0.29 \mathrm{~h}^{-1}\right)$. Nevertheless, no inhibitory effects were observed in the cellular activity under high air pressures of 3 and 5 bar. These results confirmed the ability of the yeast to cope with oxidative stress conditions that can arise from the air pressure increase, since air pressure at 5 bar is less harmful than other oxidant agents and cells were able to induce their antioxidant defenses.
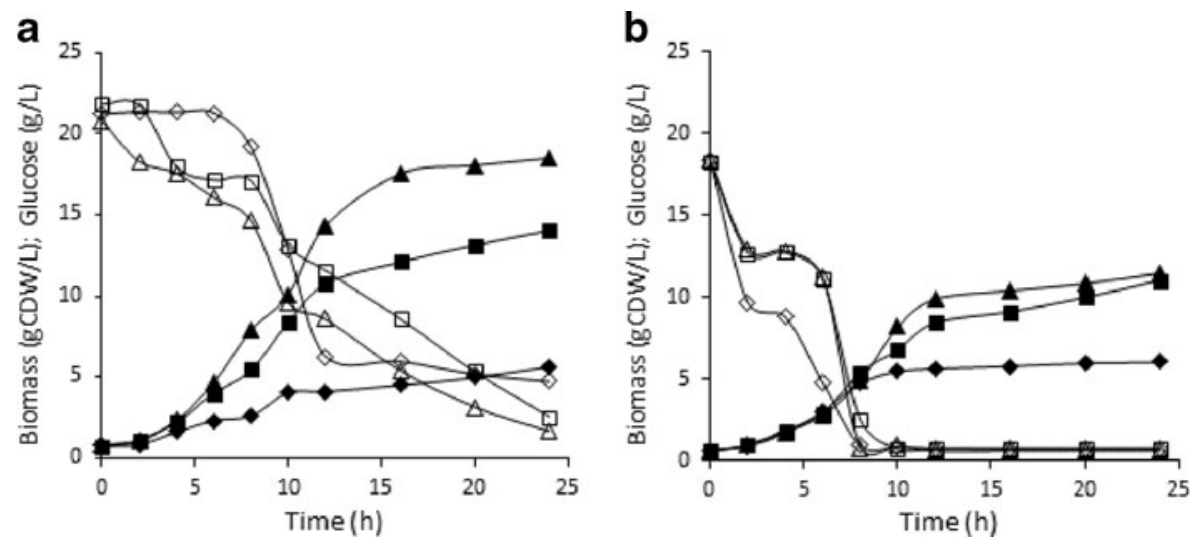

Fig. 6 Batch growth (close symbols) and glucose consumption (open symbols) of Y. lipolytica (a) and P. pastoris

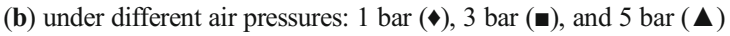




\section{Conclusions}

Under normal physiological conditions, the toxic effects of ROS are minimized by enzymatic and nonenzymatic antioxidants. However, under stressful conditions, oxidant levels may increase to overwhelm the antioxidants, resulting in cell damage.

Our results suggest that $Y$. lipolytica have a more potent antioxidant system than P. pastoris, which was proved by the higher cell viability and enzymatic mechanisms induction. Moreover, the results herein reported demonstrated that both strains were more susceptible to oxidant agents resuspended in PBS buffer than in YPD medium.

Cell responses against both superoxide and peroxide stresses include enhanced expression of SOD and catalase, which are key enzymes for direct ROS scavenging. Under superoxide stress (PQ and air pressure), the SOD induction was the main observed mechanism. In contrast, and as expected, the effect of $\mathrm{H}_{2} \mathrm{O}_{2}$ treatment on antioxidant enzyme synthesis was much more pronounced for catalase than for SOD.

The results reported herein proved that an air pressure raise up to 5 bar can be successfully applied to the batch cultivation of both $Y$. lipolytica and $P$. pastoris strains with increased biomass yields. Moreover, the induction of antioxidant defenses against the superoxide anion, demonstrated that yeast strains can cope with possible oxidative stress caused by increased air pressure. These two combined results, offer an opportunity to perform industrial bioprocesses based in these yeast strains, under increased air pressure.

Acknowledgments The authors acknowledge the financial support provided by "Fundação para a Ciência e Tecnologia” (Grant SFRH/BD/47371/2008).

\section{References}

1. Esterbauer, H., Schaur, R. J., \& Zollner, H. (1991). Free Radical Biology \& Medicine, 11, 81-128.

2. Giller, G., \& Sigler, K. (1995). Folia Microbiologica, 40(2), 131-152.

3. Joaquim, A. F. V., Francisco, P. M., Ludovina, L., \& Madeira, M. C. V. (2001). Journal of Biochemical and Molecular Toxicology, 15, 322-330.

4. 1122Davies, J. M. S., Lowry, C. V., \& Davies, K. J. A. (1995). Archives of Biochemistry and Biophysics, 317, $1-6$.

5. Belo, I., Pinheiro, R., \& Mota, M. (2005). Journal of Biotechnology, 115, 397-404.

6. Lopes, M., Gomes, N., Mota, M., \& Belo, I. (2009). Applied Biochemistry and Biotechnology, 159(1), 46-53.

7. Pinheiro, R., Belo, I., \& Mota, M. (2003). Letters in Applied Microbiology, 37, 438-442.

8. Coelho, M. A. Z., Amaral, P. F. F., \& Belo, I. (2010). Applied Biochemistry and Biotechnology, 930-944.

9. Zhang, J.-h., Wu, D., Chen, J., \& Wu, J. (2011). Biotechnology and Bioprocess Engineering, 16, 1196-1200.

10. Wang, K., Li, G., Yu, S. Q., Zhang, C. T., \& Liu, Y. H. (2010). Applied Microbiology and Biotechnology, $88,155-165$.

11. Oliveira, C., Felix, W., Moreira, R. A., Teixeira, J. A., \& Domingues, L. (2008). Protein Expression and Purification, 60, 188-193.

12. Potvin, G., Ahmad, A., \& Zhang, Z. (2012). Biochemical Engineering Journal, 64, 91-105.

13. Jones, R.-P. (1987). Process Biochemistry, 118-128.

14. Pinheiro, R., Belo, I., \& Mota, M. (2000). Enzyme and Microbial Technology, 26, 756-762.

15. Beers, R. F., \& Sizer, I. W. (1952). Journal of Biological Chemistry, 195, 276-287.

16. Marklund, S., \& Marklund, G. (1974). European Journal of Biochemistry, 47, 469-474.

17. Smith, I., Viertheller, T., \& Thorne, C. (1988). Analytical Biochemistry, 175, 408-413.

18. Espindola, A. S., Gomes, D. S., Panek, A. D., \& Eleutherio, E. A. (2003). Cryobiology, 47, $236-241$.

19. Jamnik, P., \& Raspor, P. (2003). Journal of Biochemistry and Molecular Biology, 17(6), 316-323.

20. Hassan, H. M., \& Fridovich, I. (1978). Journal of Biological Chemistry, 253(22), 8143-8148.

21. Pinheiro, R., Belo, I., \& Mota, M. (2002). Applied Microbiology and Biotechnology, 58, 842-847. 
22. Ghazi-Khansari, M., Mohammadi-bardbori, A., \& Hosseini, M. J. (2006). Annals of the New York Academy of Sciences, 1090, 98-107.

23. Grant, C. M., MacIver, F. H., \& Dawes, I. W. (1996). Current Genetics, 29, 511-515.

24. Izawa, S., Inoue, Y., \& Kimura, A. (1995). FEBS Letters, 368, 73-76.

25. Pigeolet, E., Corbisier, P., Houbion, A., Lambert, D., Michiels, C., Raes, M., et al. (1990). Mechanisms of Ageing and Development, 51, 283-297.

26. Lushchak, V., Semchyshyn, H., Lushchak, O., \& Mandryk, S. (2005). Biochemical and Biophysical Research Communications, 338, 1739-1744.

27. Abbeg, M. A., Alabarse, P. V. G., Casanova, A., Hoscheid, J., Salomon, T. B., Hackenhaar, F. S., et al. (2010). Mycopathologia, 170, 11-20.

28. Biryukova, E. N., Medentsev, A. G., Arinbasarova, A. Y., \& Akimenko, V. K. (2006). Microbiology, 75(3), 243-247.

29. Braconi, D., Possenti, S., Laschi, M., Geminiani, M., Lusini, P., Bernardini, G., et al. (2008). Journal of Agricultural and Food Chemistry, 56, 3836-3845.

30. Lushchak, V. I. (2011). Comparative Biochemistry and Physiology. C, 153, 175-190.

31. Semchyshyn, H. M., \& Lozinska, L. M. (2012). FEMS Yeast Research, 12, 761-773.

32. González-Párraga, P., Hernández, J. A., \& Argüelles, J. C. (2003). Yeast, 20, 1161-1169.

33. Kreiner, M., Harvey, L. M., \& McNeil, B. (2002). Enzyme and Microbial Technology, 30, 346-353.

34. Bayliak, M. M., Semchyshyn, H. M., \& Lushchak, V. I. (2006). Biochemistry (Moscow), 71, 1013-1020. 